J. Dairy Sci. 98:4131-4138

http://dx.doi.org/10.3168/jds.2014-9170

(C) American Dairy Science Association ${ }^{\circledR}$, 2015. Open access under CC BY-NC-ND license.

\title{
How imputation errors bias genomic predictions
}

\author{
E. C. G. Pimentel, ${ }^{1}$ C. Edel, R. Emmerling, and K.-U. Götz \\ Institute of Animal Breeding, Bavarian State Research Center for Agriculture, Grub 85586, Germany
}

\begin{abstract}
The objective of this study was to investigate in detail the biasing effects of imputation errors on genomic predictions. Direct genomic values (DGV) of 3,494 Brown Swiss selection candidates for 37 production and conformation traits were predicted using either their observed $50 \mathrm{~K}$ genotypes or their $50 \mathrm{~K}$ genotypes imputed from a mimicked $6 \mathrm{~K}$ chip. Changes in DGV caused by imputation errors were shown to be systematic. The DGV of top animals were, on average, underestimated and that of bottom animals were, on average, overestimated when imputed genotypes were used instead of observed genotypes. This pattern might be explained by the fact that imputation algorithms will usually suggest the most frequent haplotype from the sample whenever a haplotype cannot be determined unambiguously. That was empirically shown to cause an advantage for the bottom animals and a disadvantage for the top animals.
\end{abstract}

Key words: allele frequency, bias, haplotype, single nucleotide polymorphism (SNP) effect

\section{INTRODUCTION}

In recent years, the number of genotyping platforms with different SNP densities has increased considerably. Additionally, custom chips containing any desired number of SNP defined by the customer are now commercially available. These increasing possibilities with respect to marker density make the role of imputation from one panel to another important. Many studies have been conducted on the effect of imputation on genomic predictions and their reliabilities, but results reported so far are usually given in terms of overall correlations between genomic predictions from observed and imputed genotypes (e.g., Dassonneville et al., 2011; Segelke et al., 2012). A closer inspection of the consequences of imputation errors on genomic predictions might be of interest. Therefore, the objective of this

Received November 28, 2014.

Accepted February 20, 2015.

${ }^{1}$ Corresponding author: Eduardo.Pimentel@LfL.bayern.de study was to analyze to what extent imputation errors affect genomic breeding values and to investigate whether the differences in predictions caused by imputation errors follow any systematic pattern.

\section{MATERIALS AND METHODS}

Brown Swiss data from the December 2013 run of the official German-Austrian joint genomic evaluation were used. The pool of genotyped animals included 3,494 selection candidates; that is, animals without insemination bull status and that do not contribute phenotypes to the system. Routine evaluations are based on the Illumina Bovine SNP50 BeadChip (Illumina Inc., San Diego, CA). After the usual edits (i.e., exclusion of markers with call-rate $<0.95$, minor allele frequency $<0.02$, significant deviation from Hardy-Weinberg equilibrium or redundancy with another locus), 37,653 markers remained for further analyses. Detailed descriptions of the major steps, the criteria used for marker editing, and the statistical method routinely used in the German-Austrian genomic evaluation can be found in Edel et al. (2011) and Ertl et al. (2014). In brief, the statistical model is

$$
\mathbf{y}=\mu+\mathbf{D g}+\mathbf{e}
$$

where $\mathbf{y}$ is an $(n \times 1)$ vector of phenotypes of the calibration animals; that is, AI bulls contributing both genotypic and phenotypic information to the system; $\mu$ is an overall mean; $\mathrm{g}$ is a $(p \times 1)$ vector of direct genomic values (DGV), with $p=n+m$, and $m$ being the number of selection candidates; $\mathbf{D}$ is an $(n \times p)$ design matrix relating phenotypes to $\mathrm{DGV}$; and $\mathbf{e}$ is an $(n \times 1)$ vector of residuals. The variance of $\mathbf{y}(\mathbf{V})$ is assumed to be

$$
\mathbf{V}=\mathbf{D G D}^{\prime} \sigma_{a}^{2}+\mathbf{R}
$$

where $\mathbf{G}$ is a $(p \times p)$ genomic relationship matrix, $\sigma_{a}^{2}$ is the additive genetic variance, and $\mathbf{R}$ is a diagonal matrix of order $n$, elements of which are functions of the residual variance and the reliability of the corresponding phenotype (for details, see Edel et al., 2009). Matrix 
G was computed following the first method described by VanRaden (2008) as follows:

$$
\mathbf{G}=\mathbf{Z Z} \mathbf{Z}^{\prime}\left[2 \sum_{i=1}^{l} q_{i}\left(1-q_{i}\right)\right]^{-1},
$$

where $l$ is the number of markers; $q_{i}$ is the base allele frequency at locus $i$; and $\mathbf{Z}$ is a $(p \times l)$ matrix, calculated as $\mathbf{Z}=\mathbf{M}-\mathbf{Q}$, where $\mathbf{M}$ is the matrix of genotypes (coded as $-1,0$, or 1 ) and $\mathbf{Q}$ is a matrix of which the $i$ th column is $2\left(q_{i}-0.5\right)$. Predicted $\operatorname{DGV}(\hat{\mathbf{g}})$ are then calculated as

$$
\hat{\mathbf{g}}=\hat{\sigma}_{a}^{2} \mathbf{G} \mathbf{D}^{\prime} \mathbf{V}^{-1}(\mathbf{y}-\hat{\mu}) .
$$

Reliabilities of DGV are estimated from

$$
\operatorname{diag}\left\{\mathbf{G D}^{\prime} \mathbf{V}^{-1} \mathbf{D G}\right\} \text {. }
$$

Phenotypes used in the analyses were deregressed multiple across-country evaluation (MACE) proofs. Estimates of base allele frequencies were obtained using the method proposed by Gengler et al. (2007). The DGV of the selection candidates for 37 production and conformation traits were predicted using either their observed $50 \mathrm{~K}$ genotypes or their $50 \mathrm{~K}$ genotypes imputed from a $6 \mathrm{~K}$ chip. Genotypes of the $6 \mathrm{~K}$ chip were obtained by masking the SNP from $50 \mathrm{~K}$ that are not contained in the Illumina BovineLD BeadChip. Animals in the calibration set were all genotyped with the 50K chip. Masking of genotypes was only applied to selection candidates to depict a situation in which candidates are genotyped at low density. The number of calibration bulls varied depending on the trait and ranged from 1,001 to 5,390, with an average of 3,438 . Imputation was done with 2 imputation software packages: findhap v2 (VanRaden et al., 2011) and FImpute (Sargolzaei et al., 2014). The number of animals with $50 \mathrm{~K}$ genotypes in the reference population used for imputation was 6,243. From the 37,653 markers that passed the routine filtering process, 908 were not annotated. Therefore, these markers are meaningless for haplotype reconstruction and were not included in the imputation step done with findhap or FImpute. Genotypes at these loci were imputed with the sample mean gene contents (i.e., mean genotypes of the 6,243 reference animals with $50 \mathrm{~K}$ genotypes) afterward.

\section{RESULTS AND DISCUSSION}

Average allele error rates, measured as the mean proportion of wrongly imputed alleles, were $1.54 \%$ with findhap and $0.85 \%$ with FImpute. Mean proportions of correctly imputed genotypes were $96.97 \%$ with findhap and $98.33 \%$ with FImpute. Mean correlation coefficients between observed and imputed genotypes were 0.976 with findhap and 0.987 with FImpute. These numbers are similar to measures of imputation success from $6 \mathrm{~K}$ to $50 \mathrm{~K}$ reported in other studies (e.g., Boichard et al., 2012; Segelke et al., 2012; Chen et al., 2014). Across traits, average overall correlations between DGV predicted with observed or imputed genotypes were 0.987 (from 0.982 to 0.993 ) with findhap and 0.992 (from 0.988 to 0.995 ) with FImpute. These numbers are similar to correlations reported in other studies (e.g., Mulder et al., 2012; Segelke et al., 2012). Despite these overall high correlations, some noticeable reranking among the top selection candidates occurred when prediction was based on imputed genotypes. Averaged across all traits, rank correlations within the top 50 candidates were 0.843 with findhap and 0.876 with FImpute. Within the top 50 candidates, we found a tendency to underestimation when DGV were predicted from imputed genotypes. Analogously, a tendency to overestimation within the bottom 50 candidates was observed. As an illustration, mean differences between DGV from observed genotypes and from genotypes imputed with findhap for the bottom 50, intermediate, and top 50 candidates (ranked according to the DVG from observed genotypes) are given in Figure 1 for 6 of the studied traits. These trends indicate that the changes in DGV caused by imputation errors follow some systematic pattern. As a possible explanation to this phenomenon, we formulated a hypothesis based on the following 3 assumptions: (1) in a simplified way, one could postulate that the top animals should have, on average, the best haplotypes, and that the bottom animals should have, on average, the worst haplotypes, with respect to their effects on the trait being considered; (2) whenever an imputation algorithm cannot determine a haplotype unambiguously, it will suggest the most frequent haplotype in the sample as replacement for the missing one; and (3) if the most frequent haplotype has a neutral effect on the trait (i.e., if its effect is the closest to the population mean compared with the effects of the other possible haplotypes), then this replacement will represent an advantage for the bottom animals and a disadvantage for the top animals.

For most of the traits, we observed a general decrease in DGV when imputed genotypes were used. This trend can be seen in Figure 1 as the slight decrease in DGV for the intermediate animals. This overall decrease can be attributed to the genetic trend that separates the group of selection candidates from the calibration group (and the reference pool of genotyped animals used for imputation). Compared with the reference group, selection 

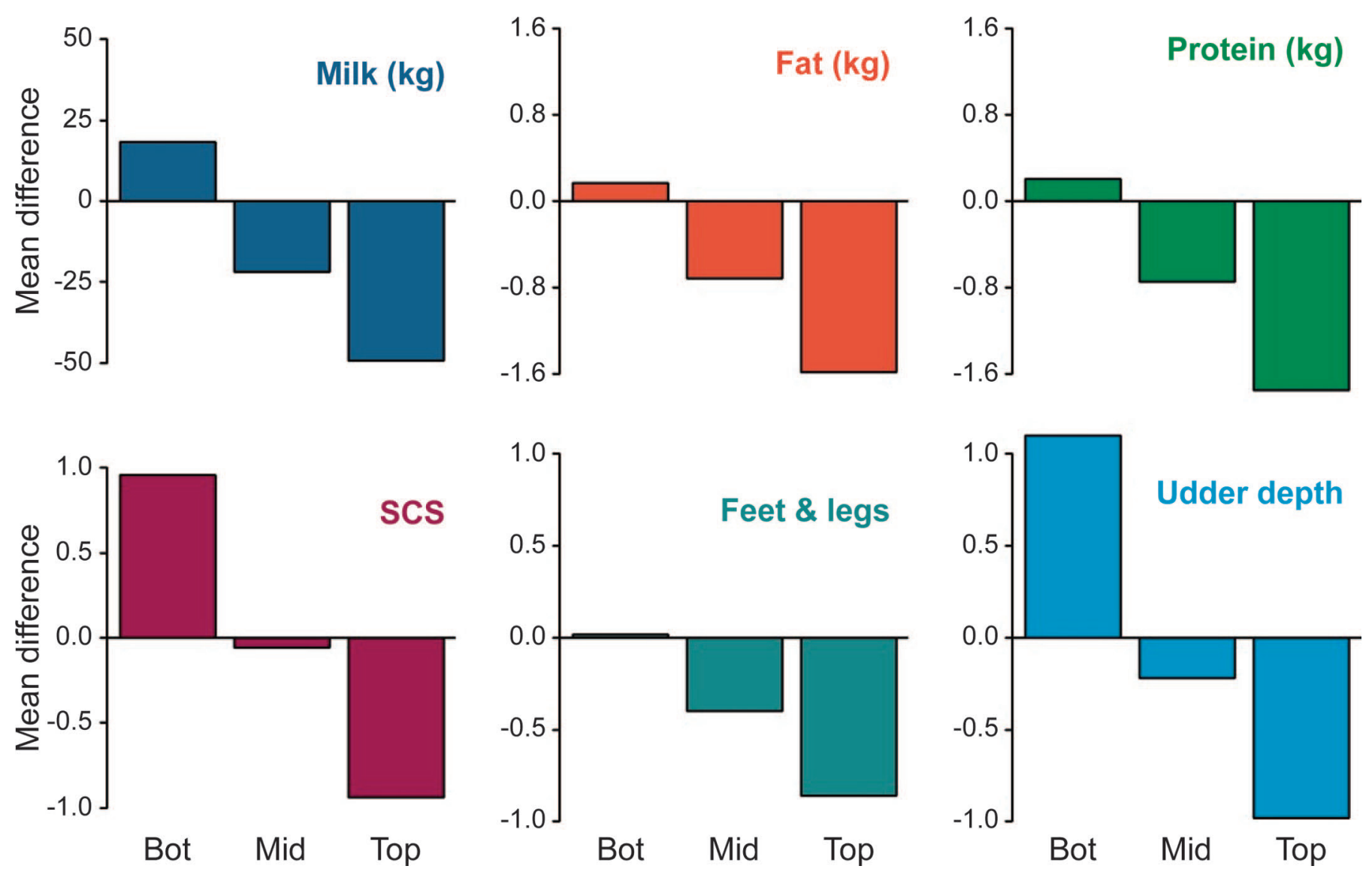

Figure 1. Mean difference between direct genomic values from imputed and from observed genotypes within class of candidate. Differences in milk, fat, and protein yields are in trait units. Differences in SCS, feet and legs, and udder depth are in points of a relative breeding value with mean 100 and standard deviation 12. Bot = bottom; Mid = middle. Color version available online.

candidates are on average better for most of the traits. This is more evident for the traits under stronger selection (e.g., milk traits and feet and legs) and less evident for the traits with lower selection intensity (e.g., udder depth and SCS). To investigate how much the mean change in DGV was consistent with the genetic trends, we calculated genetic trends for the full set of 37 traits based on the EBV of all available genotyped animals that had an EBV in the data set. Figure 2 shows the relationship between the slope of these trends (in SD of EBV per year) and the mean difference between DGV from imputed and observed genotypes (also in units of SD of EBV). This relationship fitted well a linear regression with both findhap $\left(\mathrm{R}^{2}=0.79\right)$ and FImpute $\left(\mathrm{R}^{2}=0.53\right)$. For the few traits with negative genetic trends, selection candidates were, on average, worse than the reference pool. For these traits, instead of a decrease, there was an overall increase in mean DGV with imputed genotypes (upper left part of Figure 2). These trends are in agreement with what one would expect under the hypothesis formulated above.
The analyzed data were used to calculate some statistics to investigate if the abovementioned assumptions would hold. The first assumption does not need to be addressed because the model used for predicting DGV here, and in fact the definition of breeding value in terms of average effects (Falconer and Mackay, 1996), implies exactly what was formulated in point (1). The second assumption is in agreement with the descriptions of the algorithms used in population imputation (e.g., VanRaden et al., 2011). Nevertheless, we checked in the imputed genotype data set how often an incorrectly imputed homozygous genotype was homozygous for the most frequent allele of the locus. In $73 \%$ of the cases (with both findhap and FImpute), the wrong homozygous genotype was homozygous for the most frequent allele. We also looked at differences in minor genotype frequencies and minor allele frequencies between the imputed and the original data sets. Differences were generally very small. On average, minor genotype frequencies were slightly lower with imputed genotypes, which is in agreement with the expectation under as- 


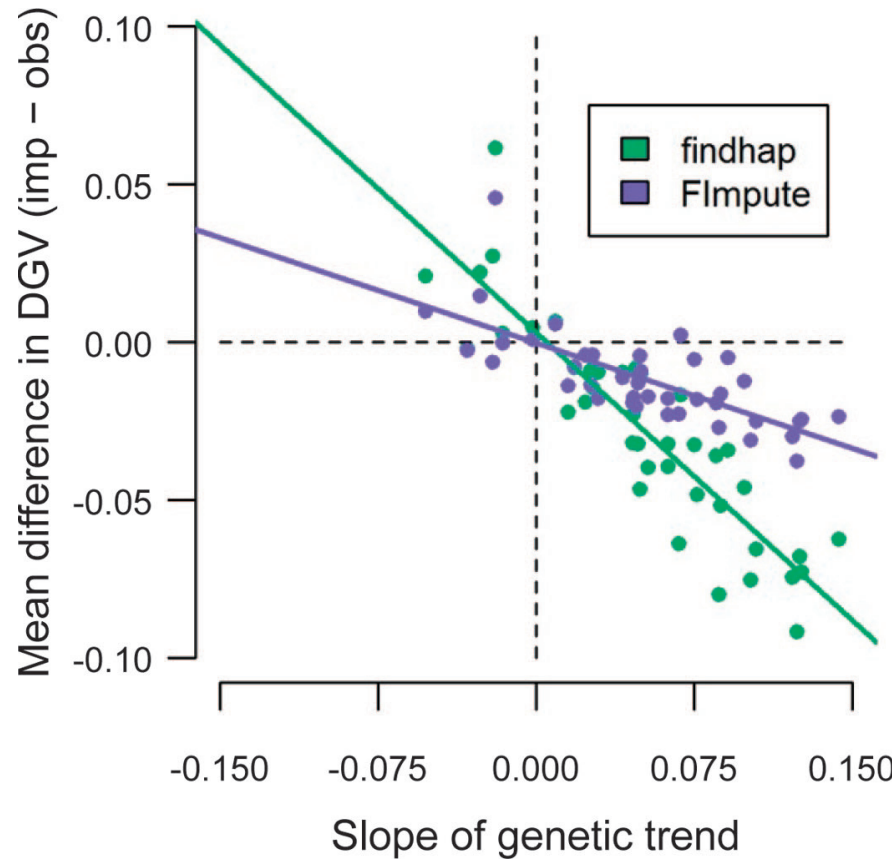

Figure 2. Mean difference (in SD of EBV) between direct genomic values (DGV) from imputed (imp) and observed (obs) genotypes against genetic trend of the trait (in SD of EBV per year); findhap (VanRaden et al., 2011); FImpute (Sargolzaei et al., 2014). Color version available online.

sumption (2). We observed that trend with both findhap and FImpute (with negligible difference). As an illustration, mean frequencies of the minor genotype and the minor allele within the observed and imputed data sets are presented in Figure 3. Minor allele frequencies also decreased slightly when imputation was done with FImpute, which agrees with the assumption made in point (2). With findhap, despite the average decrease in minor genotype frequency, we observed an average increase in minor allele frequency. We then looked at the frequencies of heterozygous genotypes in the original and imputed data sets and observed an increase in the frequency of heterozygotes when genotypes were imputed with findhap (Figure 3), which explains the increase in minor allele frequency.

To check the relationship between the effect of a given allele and its frequency in the population we used quantitative genetics theory (Falconer and Mackay, 1996). Let us assume a biallelic locus with alleles $A_{1}$ and $\mathrm{A}_{2}$, and allele frequencies $p$ and $q$, respectively. Genotypic values are $-a$ for the genotype $\mathrm{A}_{2} \mathrm{~A}_{2}$ and $+a$ for the genotype $A_{1} A_{1}$, and the dominance deviation is $d$ for the heterozygous genotype. Under the assumption of Hardy-Weinberg equilibrium, the population mean is given by $M=a(p-q)+2 p q d$. The average effects of alleles $\mathrm{A}_{1}$ and $\mathrm{A}_{2}$, expressed as a deviation from the population mean, are then given by the following equations: $\alpha_{1}=q[a+d(q-p)]$ and $\alpha_{2}=-p[a+d(q-p)]$.

We calculated the average effect of allele $\mathrm{A}_{1}$ for different values of its frequency $p$ ranging from 0 to 1 . We fixed the value of $a$ at 1 and considered 4 different scenarios with respect to the dominance deviation; namely, no dominance $(d=0)$, some dominance $(d=$ $0.5)$, complete dominance $(d=1)$, and overdominance $(d=1.5)$. Results are given in Figure 4 . For all 4 scenarios, we see that the higher the frequency of allele $\mathrm{A}_{1}$ in the population, the smaller the deviation of its average effect from the population mean. This is in agreement with the assumption made in the first part of point (3). To further investigate if the third assumption might hold, we looked at the SNP effects on each of the analyzed traits and checked whether an incorrectly imputed allele had an increasing or decreasing
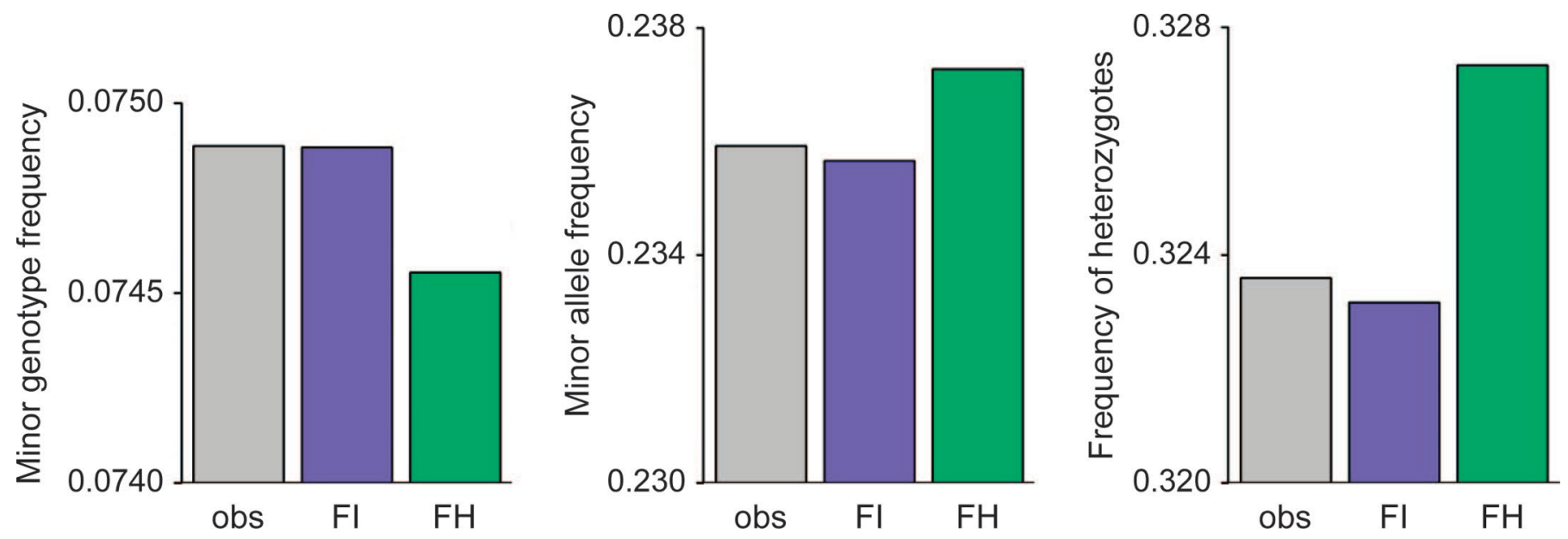

Figure 3. Mean frequencies of minor genotype, minor allele, and heterozygous genotype in the observed (obs) and imputed [FI $=$ FImpute (Sargolzaei et al., 2014) and FH = findhap (VanRaden et al., 2011)] data sets. Color version available online. 
effect on the breeding value. For each trait, imputation software, and group, the mean proportions of wrong replacements for an allele with a positive or a negative effect on the trait were computed. Results for the top and bottom groups with FImpute are given in Figure 5 . One can see that within the group of top candidates (uppermost part of the figure), changes for an allele with a negative effect on the trait occurred more often than changes for an allele with a positive effect. This phenomenon is expected if the above assumptions hold. For instance, when the rare allele is favorable, we would expect that most animals having this rare allele are in the top group. Because this allele is rare, imputation algorithms will rather suggest the other frequent neutral allele (unfavorable compared with the rare) as replacement. So we see these disadvantageous replacements occurring more often in the top group. Most bottom animals should already have the most frequent allele in this case, so it is seldom replaced. Analogously, the lower part of Figure 5 shows that, within the group of bottom candidates, changes occurred more often to an allele with a positive effect. Following the same argument, when the rare allele is unfavorable, the bottom animals should be the ones having it. Imputation would replace it by the frequent neutral allele (favorable compared with the rare), bringing an advantage for the bottom animals. In that case, top animals should already have the favorable frequent allele, so these advantageous replacements do not occur so frequently in the top group. These patterns were similar for both findhap and FImpute and are in agreement with the assumption made in point (3). The statistics calculated from the analyzed data are in good agreement with expectations if the assumptions made above were correct. As mentioned before, unannotated markers were imputed with the sample mean gene contents, which might have contributed to the observed agreement. We calculated these statistics again, leaving the unannotated markers out of the calculation, and observed the same trends depicted above. This does not prove the formulated hypothesis but gives strong empirical evidence that it may hold.

Because imputed alleles will often be the ones at higher frequency in the sample (at least whenever they cannot be unambiguously determined from genotype information on relatives), imputation errors are expected to reduce variation in genomic relationships. To check this with our data, we computed genomic relationship matrices comprising the selection candidates using either observed or imputed genotypes. As previously mentioned, matrices were set up following the first procedure described in VanRaden (2008). Standard deviation of off-diagonal elements were 0.054 with observed and 0.052 with imputed genotypes.
Variation of diagonal elements also decreased slightly with imputed genotypes, with standard deviation going from 0.028 to 0.027 . There was little difference between the standard deviations from findhap and FImpute. Differences in genomic relationships from observed and imputed genotypes were minimal but there was a noticeable change in genomic inbreeding coefficients when imputed genotypes were used in the computation (Figure 6). Average genomic inbreeding was $8.57 \%$ with observed genotypes, $5.71 \%$ with FImpute, and $5.57 \%$ with findhap. Interestingly, the amount of heterozygosity in the observed and imputed data sets was quite similar (Figure 7). Average proportions of heterozygosity of selection candidates were $33.93 \%$ with observed genotypes, $33.89 \%$ with FImpute, and $34.24 \%$ with findhap. This reduction in genomic inbreeding without a corresponding increase in heterozygosity could be explained by the way genomic inbreeding was calculated. In the computation of genomic inbreeding coefficients, a larger weight is put on homozygous loci for rare alleles (VanRaden, 2008), which are less likely to occur with imputed genotypes because imputation algorithms will suggest more-frequent alleles. These observations also ratify the feature of imputation algorithms noted in assumption (2).

Another aspect worth reporting is the difference in reliability between DGV from observed and imputed

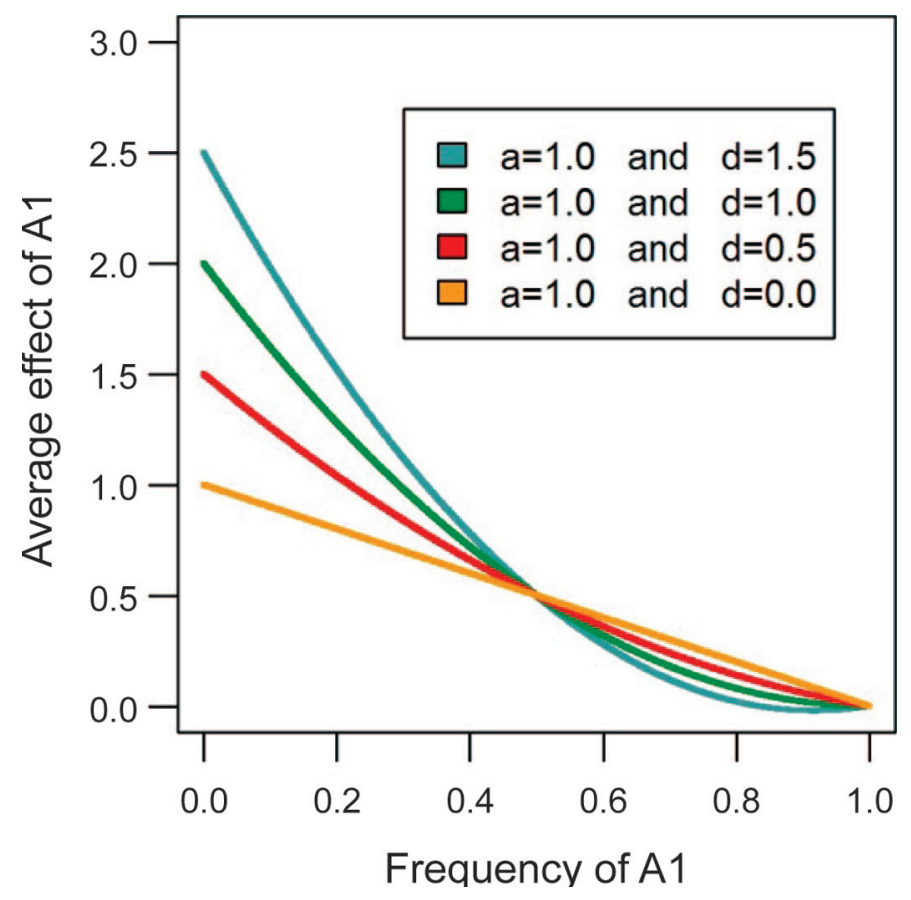

Figure 4. Average effect of an allele (expressed as a deviation from the population mean) in dependence of its frequency, for a given genotypic value $(a)$ and dominance deviation $(d)$. Color version available online. 

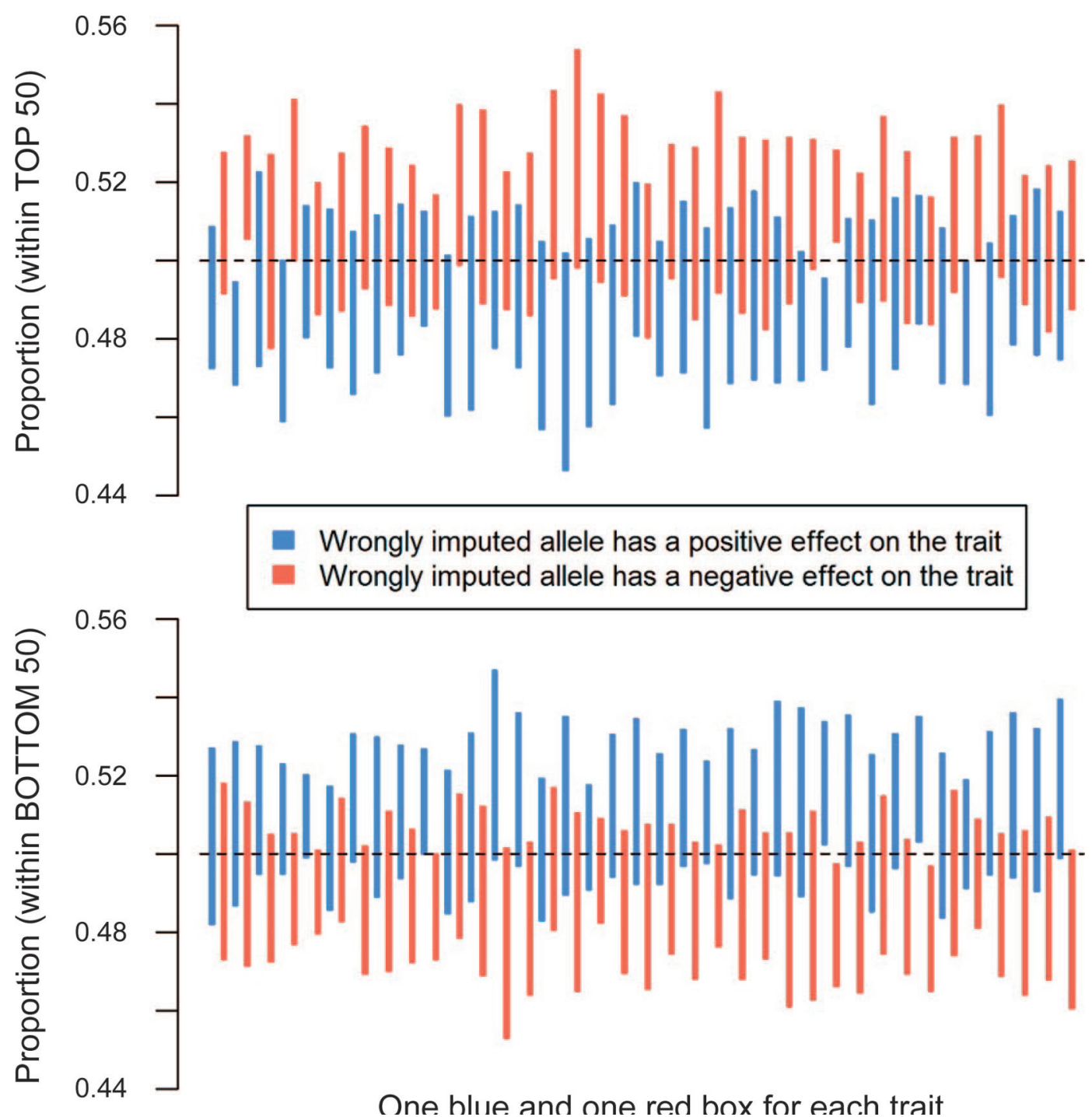

Figure 5. Boxplots (from first to third quartile, without whiskers) of the proportion of wrongly imputed alleles that have a positive (or a negative) effect on the trait. Color version available online.

genotypes. One could argue that the reported underand overestimation may be interpreted as decreased variation in DGV due to reduced reliability of DGV from imputed genotypes. This argument is related to the hypothesis formulated here. By assigning common haplotypes observed in the sample, imputation removes genetic variance from the data. Evidence of this reduction in genetic variance was reported above in the form of lower standard deviations observed in genomic relationships from imputed genotypes, and especially in the form of considerably lower genomic inbreeding coefficients. Lower genetic variance induced lower reliabilities of predicted DGV. In all traits studied here, we observed a decrease in reliability when imputed genotypes were used for predicting DGV. Across traits, mean $( \pm \mathrm{SD})$ differences between reliabilities of DGV from imputed and from observed genotypes were -1.91 \pm 0.53 with findhap and $-1.17 \pm 0.46$ with FImpute. The decrease in reliability should be directly related to the amount of imputation errors. Figure 8 illustrates the relationship between mean imputation accuracy (correlation between observed and imputed genotypes) and the decrease in reliability of DGV from genotypes imputed with findhap for 6 traits.

VanRaden et al. (2013) compared genomic evaluations with $50 \mathrm{~K}$ and with high-density (HD) chips and reported that, for many traits, the largest SNP effects with HD were for the same 50K markers. They argued that this might be caused by imputation errors. Their findings and argument are consistent with our observa- 


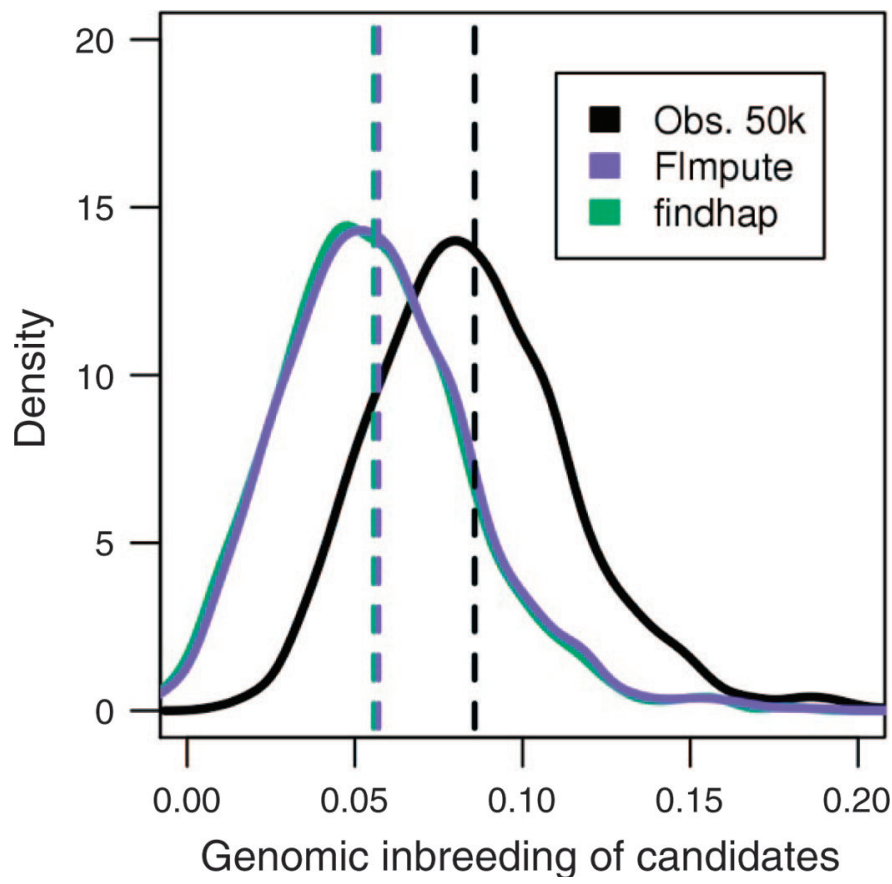

Figure 6. Distributions of genomic inbreeding coefficients of selection candidates computed from observed (Obs. 50k) or from genotypes imputed with FImpute (Sargolzaei et al., 2014) or findhap (VanRaden et al., 2011). Color version available online.

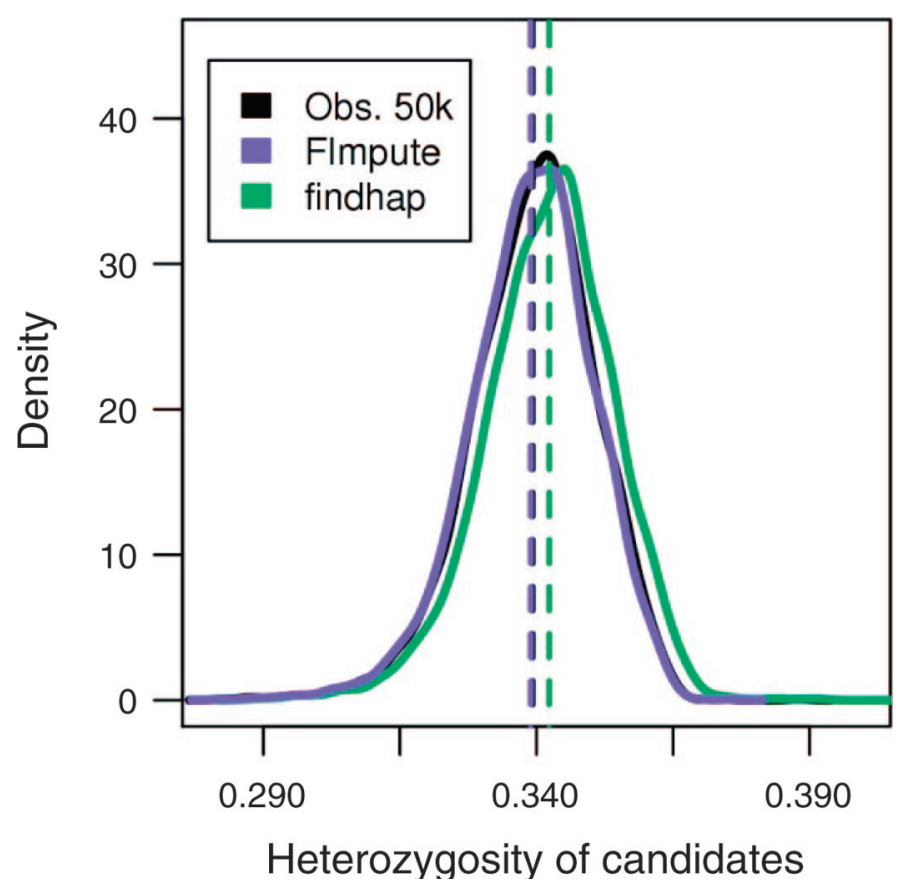

Figure 7. Distributions of the proportions of heterozygosity of selection candidates computed from observed (Obs. 50k) or from genotypes imputed with FImpute (Sargolzaei et al., 2014) or findhap (VanRaden et al., 2011). Color version available online.

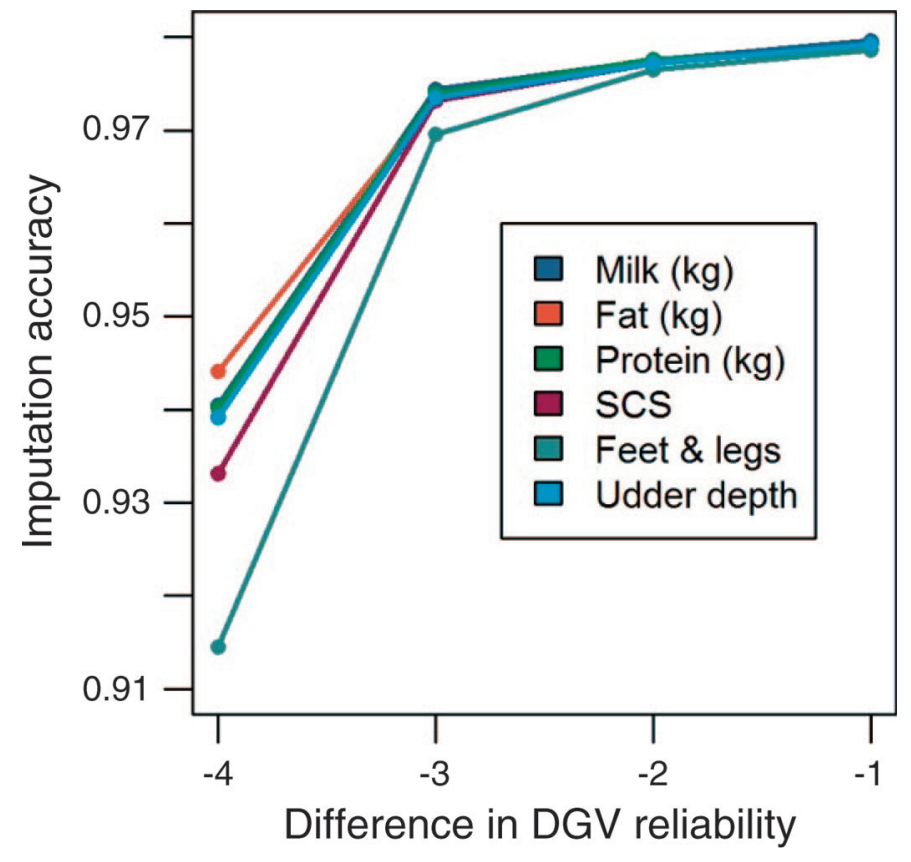

Figure 8. Mean imputation accuracy within level of reduction in direct genomic value (DGV) reliability from genotypes imputed with findhap (VanRaden et al., 2011) compared with reliabilities from observed genotypes. Color version available online.

tion that imputation algorithms often suggest markers at highest frequencies, which should have moderate or neutral effects. This could explain why HD imputed loci did not show up with largest effects for many traits in the study of VanRaden et al. (2013). These features of imputation algorithms also have an effect on statistical power to detect significant regions underlying complex phenotypes in association studies. Huang et al. (2009) investigated the relationship between imputation errors and power in association studies and suggested that each $1 \%$ increase in imputation error would require an increase of 5 to $13 \%$ in sample size to maintain power.

\section{CONCLUSIONS}

In conclusion, imputation errors seem to cause systematic changes in genomic predictions, which tend to be underestimated in the top segment and overestimated in the bottom segment. This pattern might be explained by the fact that imputation algorithms will usually suggest the most frequent haplotype observed in the sample as replacement for the missing one whenever a haplotype cannot be determined unambiguously. This feature of imputation was empirically shown to give an advantage to animals in the bottom and a disadvantage to animals in the top segment. That might have implications in genomic evaluations, especially with data pools comprising animals genotyped at 
different densities and strong selection. In such cases, good selection candidates genotyped using low-density panels could be penalized.

\section{ACKNOWLEDGMENTS}

We gratefully acknowledge the Arbeitsgemeinschaft Süddeutscher Rinderzucht- und Besamungsorganisationen e.V. (Munich, Germany) for their financial support within the research cooperation "Zukunftswege." Many of the genotypes used in this study were obtained through our participation in the InterGenomics project. We thank Paul VanRaden (Animal Genomics and Improvement Laboratory, Beltsville, MD) for making findhap freely available online, Mehdi Sargolzaei (Boviteq Alliance \& CGIL, University of Guelph, Guelph, ON, Canada) for kindly providing a trial version of FImpute, and two anonymous reviewers for fruitful suggestions.

\section{REFERENCES}

Boichard, D., H. Chung, R. Dassonneville, X. David, A. Eggen, S. Fritz, K. J. Gietzen, B. J. Hayes, C. T. Lawley, T. S. Sonstegard, C. P. Van Tassell, P. M. VanRaden, K. A. Viaud-Martinez, and G. R. Wiggans. 2012. Design of a bovine low-density SNP array optimized for imputation. PLoS ONE 7:e34130.

Chen, L., C. Li, M. Sargolzaei, and F. Schenkel. 2014. Impact of genotype imputation on the performance of GBLUP and Bayesian methods for genomic prediction. PLoS ONE 9:e101544.

Dassonneville, R., R. F. Brøndum, T. Druet, S. Fritz, F. Guillaume, B. Guldbrandtsen, M. S. Lund, V. Ducrocq, and G. Su. 2011. Effect of imputing markers from a low-density chip on the reliability of genomic breeding values in Holstein populations. J. Dairy Sci. 94:3679-3686.
Edel, C., R. Emmerling, and K.-U. Götz. 2009. Optimized aggregation of phenotypes for MA-BLUP evaluation in German Fleckvieh. Interbull Bull. 40:178-183.

Edel, C., H. Schwarzenbacher, H. Hamann, S. Neuner, R. Emmerling, and K.-U. Götz. 2011. The German-Austrian genomic evaluation system for Fleckvieh (Simmental) cattle. Interbull Bull. 44:152156.

Ertl, J., C. Edel, R. Emmerling, H. Pausch, R. Fries, and K.-U. Götz. 2014. On the limited increase in validation reliability using highdensity genotypes in genomic best linear unbiased prediction: Observations from Fleckvieh cattle. J. Dairy Sci. 97:487-496.

Falconer, D. S., and T. F. C. Mackay. 1996 Introduction to Quantitative Genetics. 4th ed. Pearson Longman, Harlow, UK.

Gengler, N., P. Mayeres, and M. Szydlowski. 2007. A simple method to approximate gene content in large pedigree populations: application to the myostatin gene in dual-purpose Belgian Blue cattle. Animal 1:21-28.

Huang, L., C. Wang, and N. A. Rosenberg. 2009. The relationship between imputation error and statistical power in genetic association studies in diverse populations. Am. J. Hum. Genet. 85:692-698.

Mulder, H. A., M. P. L. Calus, T. Druet, and C. Schrooten. 2012. Imputation of genotypes with low-density chips and its effect on reliability of direct genomic values in Dutch Holstein cattle. J Dairy Sci. 95:876-889.

Sargolzaei, M., J. P. Chesnais, and F. S. Schenkel. 2014. A new approach for efficient genotype imputation using information from relatives. BMC Genomics 15:478.

Segelke, D., J. Chen, Z. Liu, F. Reinhardt, G. Thaller, and R. Reents. 2012. Reliability of genomic prediction for German Holsteins using imputed genotypes from low-density chips. J. Dairy Sci. 95:5403-5411.

VanRaden, P. M. 2008. Efficient methods to compute genomic predictions. J. Dairy Sci. 91:4414-4423.

VanRaden, P. M. D. J. Null, M. Sargolzaei, G. R. Wiggans, M. E. Tooker, J. B. Cole, T. S. Sonstegard, M. A. Faust, and G. A. Doak 2013. Genomic imputation and evaluation using high-density Holstein genotypes. J. Dairy Sci. 96:668-678.

VanRaden, P. M., J. R. O'Connell, G. R. Wiggans, and K. A. Weigel. 2011. Genomic evaluations with many more genotypes. Genet. Sel. Evol. 43:10. 